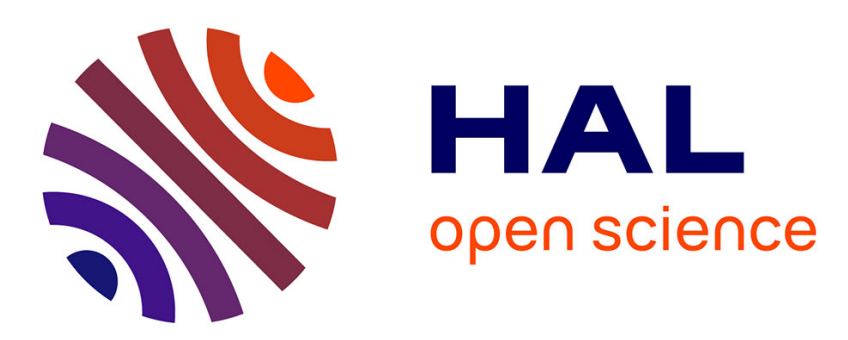

\title{
A novel spatial and stochastic model to evaluate the within and between farm transmission of classical swine fever virus: I. General concepts and description of the model
}

\author{
B. Martínez-López, B. Ivorra, A.M. Ramos, J.M. Sánchez-Vizcaíno
}

\section{To cite this version:}

B. Martínez-López, B. Ivorra, A.M. Ramos, J.M. Sánchez-Vizcaíno. A novel spatial and stochastic model to evaluate the within and between farm transmission of classical swine fever virus: I. General concepts and description of the model. Veterinary Microbiology, 2010, 147 (3-4), pp.300. 10.1016/j.vetmic.2010.07.009 . hal-00654950

\section{HAL Id: hal-00654950 https://hal.science/hal-00654950}

Submitted on 24 Dec 2011

HAL is a multi-disciplinary open access archive for the deposit and dissemination of scientific research documents, whether they are published or not. The documents may come from teaching and research institutions in France or abroad, or from public or private research centers.
L'archive ouverte pluridisciplinaire HAL, est destinée au dépôt et à la diffusion de documents scientifiques de niveau recherche, publiés ou non, émanant des établissements d'enseignement et de recherche français ou étrangers, des laboratoires publics ou privés. 


\section{Accepted Manuscript}

Title: A novel spatial and stochastic model to evaluate the within and between farm transmission of classical swine fever virus: I. General concepts and description of the model

Authors: B. Martínez-López, B. Ivorra, A.M. Ramos, J.M. Sánchez-Vizcaíno

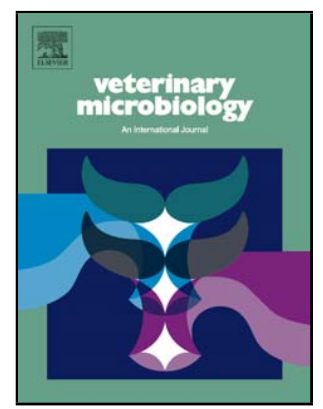

PII: S0378-1135(10)00344-5

DOI: doi:10.1016/j.vetmic.2010.07.009

Reference: VETMIC 4965

To appear in: $\quad$ VETMIC

Received date: $\quad$ 2-11-2009

Revised date: $\quad$ 7-7-2010

Accepted date: $\quad$ 15-7-2010

Please cite this article as: Martínez-López, B., Ivorra, B., Ramos, A.M., SánchezVizcaíno, J.M., A novel spatial and stochastic model to evaluate the within and between farm transmission of classical swine fever virus: I. General concepts and description of the model, Veterinary Microbiology (2010), doi:10.1016/j.vetmic.2010.07.009

This is a PDF file of an unedited manuscript that has been accepted for publication. As a service to our customers we are providing this early version of the manuscript. The manuscript will undergo copyediting, typesetting, and review of the resulting proof before it is published in its final form. Please note that during the production process errors may be discovered which could affect the content, and all legal disclaimers that apply to the journal pertain. 
1 A novel spatial and stochastic model to evaluate the within and between farm

2 transmission of classical swine fever virus: I. General concepts and description of the

3 model.

4

5 Martínez-López, B..$^{\text {a* }}$; Ivorra, B. ${ }^{\text {b }}$; Ramos, A.M. ${ }^{\text {b }}$; Sánchez-Vizcaíno, J. M. ${ }^{\text {a }}$

6

$7 \quad{ }^{a}$ VISAVET, Animal Health Department. Veterinary School. Complutense University of

8 Madrid. Av. Puerta de Hierro s/n. 28040, Madrid, Spain

$9{ }^{\mathrm{b}}$ Applied Mathematics Department. Mathematics School. Complutense University of

10 Madrid. Plaza de Ciencias 3, 28040, Madrid, Spain.

$11{ }^{*}$ Corresponding author: Tel: +34 9139437 02; Fax: +34 9139439 08; E-mail address:

12 beatriz@sanidadanimal.info; Av. Puerta de Hierro s/n. 28040, Madrid, Spain. 


\section{Abstract}

A new stochastic and spatial model was developed to evaluate the potential spread of

17 classical swine fever virus (CSFV) within and between farms, and considering the specific

18 farm-to-farm contact network. Within-farm transmission was simulated using a modified SI

19 model. Between-farm transmission was assumed to occur by direct contacts (i.e. animal movement) and indirect contacts (i.e. local spread, vehicle and person contacts) and considering the spatial location of farms. Control measures dictated by the European legislation (i.e. depopulation of infected farms, movement restriction, zoning, surveillance, contact tracing) were also implemented into the model. Model experimentation was performed using real data from Segovia, one of the provinces with highest density of pigs in Spain, and results were presented using the mean, $95 \%$ probability intervals [95\% $\mathrm{PI}]$ and risk maps. The estimated mean $[95 \% \mathrm{PI}]$ number of infected, quarantined and depopulated farms were $3[1,17], 23[0,76]$ and $115[0,318]$, respectively. The duration of the epidemic was $63[26,177]$ days and the most important way of transmission was associated with local spread (61.4\% of the infections). Results were consistent with the spread of previous CSFV introductions into the study region. The model and results presented here may be useful for the decision making process and for the improvement of the prevention and control programmes for CSFV.

34 Keywords: classical swine fever; simulation model; disease spread; stochastic; decision tool

\section{Introduction}


Classical swine fever (CSF) is a highly contagious viral disease of domestic and wild pigs which generates important economical losses in the affected regions. The disease is caused by the CSF virus (CSFV), a Pestivirus belonging to the Flaviviridae family. Despite the efforts to control and eradicate CSF, this disease remains endemic in many countries of South and Central America, Africa and South-east of Asia and sporadic outbreaks have been affecting 57\% of the European countries from 1996 to 2008 (OIE, 2009a,b).

During a CSF epidemic, the spread of CSFV from an infected animal to other susceptible ones may occur either by direct or indirect contacts. Direct transmission implies animal-to-animal effective contact from an infectious animal to a susceptible one. Indirect transmission requires an effective contact between a contaminated fomite (i.e. vehicle, insect, material or people) and a susceptible animal. Historically, the movement of infected animals and contaminated vehicles has been reported as one of the main routes of CSFV spread, although other routes such as airborne spread, movement of people, use of infected semen or feed, iatrogenic infection and mechanical vectors such as rodents, insects, birds or pets has also been described as potential ways of transmission (De Vos et al., 2003). Nevertheless, the spread patterns and magnitude and duration of a CSF epidemic change depending on the epidemiological and demographic characteristics of the infected region and the timing and effectiveness of the control measures applied. For this reason, it is difficult to extrapolate the routes of infection and consequences of a CSF epidemic from one region to another.

The study of the potential spread patterns of CSFV into a region may help to identify risk factors for disease spread and to improve the prevention and management of future outbreaks. In CSFV-free areas, the only way to quantify the magnitude of potential epidemics and evaluate the effectiveness of different control measures to be applied is to use epidemiologic and mathematic models. These models are intended to simulate the potential spread of CSFV into a region in order to evaluate effective measures to control disease and have a decision 
support system to better manage real outbreaks. Recently, some models have been developed

64

65

66

67

68 to simulate the potential spread of CSFV into free regions such as Belgium (Staatkamp et al., 1996; Ribbens et al., 2007), Germany (Stärk, 1998; Karsten et al., 2005a,b), The Netherlands (Stegeman et al., 1999; Jalvingh et al., 1999) and Australia (Milne et al., 2008). Martinez el al. (2009) also described a spatial stochastic model for Spain by using a commercial available software (InterSpread Plus, Sanson et al. 1993). However, most of these models only focus on the between-farm transmission of the CSFV, with poor assumptions regarding within-farm transmission and, do not explicitly consider the specific farm-to-farm contact network into the study region.

The study presented here, is intended to provide quantitative estimates of the magnitude and duration of potential CSF-epidemics by using a stochastic and spatial model to simulate both the within and between farm transmission and considering the specific farm-to-farm contacts. The model would provide a decision-support system and results of this study will be useful in the development of prevention and control strategies to better manage future CSFV outbreaks.

\section{Material and Methods}

\subsection{General description of the model}

A spatial stochastic model, referred to as Be-FAST (Between -Farm -Animal Spatial Transmission) Disease Model was developed to evaluate the daily spread of CSFV within and between farms into a specific region.

At the beginning of the simulation $(\mathrm{t}=0)$ all farms were in the susceptible state (i.e. non infected) except a randomly selected farm, called index case, which was assumed to have one infected pig and was classified as infected. Then, during a time interval $[0, T]$, the within- 
and between- farm daily CSFV-transmission process was simulated following the steps described in sections 2.3 and 2.4. The process of random selection of the index case was repeated 1,000 times to generate 1,000 different epidemics using the Monte Carlo method. Control measures based on zoning, movement restriction, depopulation of infected farms, increase of surveillance and tracing were also implemented into the model and can be activated/deactivated in order to quantify their effectiveness to reduce the magnitude and duration of the epidemic.

The main model outputs were the number of infected, quarantined and depopulated farms and duration time of the epidemic. Quantification of CSFV farm-to-farm transmission was evaluated by the computation of the risk of infection, the basic reproduction ratio and the effective reproduction ratio. Risk of infection was defined as the number of times that a farm became infected after the different simulated epidemics. Basic reproduction ratio, $R_{0}$, was defined as the expected number of secondary infections (i.e. effective contacts) arising from a single infected farm during its entire infectious period under the assumption that all other farms remain susceptible (naïve population). Effective reproduction ratio, $R$, was defined as the amount of secondary infections caused by one infected farm in a not naïve population (Anderson and May, 1991). Results were presented using mean and 95\% prediction interval (95\% PI) and visual outputs were represented using graphs, epidemic curves and risk maps.

\subsection{Farm data}

Data available for each farm, identified as farm $h$, included the geographical location of the farm $\left(X_{h}\right.$ and $Y_{h}$ coordinates of the farm centroid), the number of pigs per farm $\left(N_{h}\right)$, the type of farm $\left(T_{h}\right)$, the Integration group $\left(I N T_{h}\right)$, which refers to group of farms which share material and vehicles and, the Sanitary Defence Association group $\left(A D S_{h}\right)$ which refers to 
112 group which share private veterinarians. The type of pig farms registered was farrowing,

113 fattening, farrow-to-finish pig farms, artificial insemination centres and slaughterhouses.

114 Furthermore, pig shipments from farm-to-farm, number of pigs shipped, date of

115 shipment and, farm of origin and destination of the shipment occurring from January $1^{\text {st }}$ to

116 December 31 of 2008 was also available.

117 Information on farm location allowed to simulate the between-farm transmission,

118 accounting for the spatial heterogeneities within the study region.

119

120

\subsection{Within-farm CSFV transmission}

121

The CSFV spread within a particular pig farm $h$ was modeled using a modified state transition model, in which a pig was characterized, for the sake of simplicity ${ }^{\mathrm{a}}$, to be in one of two states either susceptible $(S p)$ or infected $(I p)$. The transition from "susceptible" to “infected" state was governed by a stochastic SI model (Anderson and May, 1979), which is activated when the farm $h$ reaches the state of "infectious $\left(T_{h}\right)$ " (see Section 2.4). The number of susceptible $S p_{h}(t)$ and infected $I p_{h}(t)$ pigs at farm $h$ at day $t$, was generated using a random

128 variable following a Poisson distribution with mean of $\beta_{h} \frac{S p_{h}(t) I p_{h}(t)}{N_{h}(t)}$ where $\beta_{h}$ is the transmission parameter set to $8.52,1.85$ or 5.18 depending if we consider farrowing, fattening 130 or farrow-to-finish pig farms, respectively (Klinkenberg et al., 2002) (Table 1).

\subsection{Between-farms CSFV transmission}

\footnotetext{
${ }^{a}$ Here, in order to reduce computational complexity, the infectious and clinical sign states were simulated at the farm level and the natural and disease mortality of pigs were assumed to be negligible.
} 

transition model with five mutually exclusive states: susceptible $\left(S_{h}\right)$, infected $\left(I_{h}\right)$, infectious $\left(T_{h}\right)$ and clinical signs $\left(C_{h}\right)$.

More precisely: a farm was assumed to be in the "susceptible" state when no pig within the farm is infected with CSFV. The "infected" state was assumed for a farm with at 138 least one infected pig (but none of them are still infectious for other pigs). The "infectious" state was assumed for those farms with at least one pig reaching the latent period. Finally the "clinical signs" state was assumed for those farms with at least one pig reaching the incubation period. Farms in either infectious or clinical signs state were assumed to be a

142 potential source of infection to other pig farms.

The order of transition from one state to another was: $S_{h} \rightarrow I_{h} \rightarrow T_{h} \rightarrow C_{h}$. The assumptions and parameters of the mathematical model regulating the transition from a particular sate to the next one are described in next subsections and table 1.

\subsubsection{Transition from "susceptible" to "infected" farm state}

The transmission of CSFV from farm-to-farm was modeled by considering both direct and indirect contacts.

Transmission by direct contacts was assumed to occur by the movement of pigs from farm $h$ to farm $k$, at day $t$.

The movement from farm-to-farm was simulated by using the animal movement network during 2008, which included 2,007,889 possible different movements. More precisely, we simulate the number of movements at day $t S M(t)$ by considering a Poisson of rate $M(t)$, where $M(t)$ is the number of movements occurring during 2008 at day $t$ (Table 1)

157 select by considering the following process. First, the number of movements $m_{h k}$ from farm $h$ 
to farm $k$ during 2008 was computed. In case of no movement between two farms during $2008, m_{h k}$ was set to $10^{-6}$ to account for movements not occurring during that particular year.

160 Finally, the probability of this movement to be selected is given by $m_{h k} / M T$ where $M T$ is the 161 total number of movements in the study region during 2008.

162 The number of pigs moved during a simulated movement occurring from farm $h$ to

163 farm $k$ at day $t$ was computed as $n_{h k} * N_{h}(t) / N_{h}(0)$ where $n_{h k}$ was the number of pigs moved during 2008 between those farms and divided by $m_{h k .}$. In case of no movement from farm $h$ to farm $k$ during $2008 n_{h k}$ was set to the mean number of moved pigs from farm $h$ during 2008 . contact depends on the number of infected pigs moved on the farm $h$ where the shipment was originated. The number of infected pigs moved from farm $h$ to $k$, is obtained by combining the number of infected pigs at time $t$ on farm $h$ where the shipment was originated, which is given by the modified SI model described in Section 2.3, and a random number given by a Poisson distribution which rate is the mean number of pigs shipped from farm $h$ to farm $k$

172 during 2008. When farm $h$ moves infected pigs to farm $k$ the number of infected pigs at farm

$173 h\left(I p_{h}\right)$ is reduced whereas the number of infected pigs at farm $k\left(I p_{k}\right)$ increases. Transmission by indirect contacts was assumed to occur by either movement of vehicles transporting animals, vehicles transporting products (feed or slurry), movement of

176 people or local spread. The number of contacts from farm-to-farm by vehicles transporting pigs was

178 simulated by using the animal movement contact network together with the simulation of 179 animal movements described above. The probability of transmission due to contact of a 180 contaminated vehicle transporting pigs was modeled using a Bernoulli distribution with mean 

only among those farms belonging to the same integration $\left(I N T_{h}\right)$ and with the assumption that a vehicle can visit a maximum of 4 farms per day (CyL expert opinion, $2008^{\mathrm{b}}$ ) and can only been infectious if previously has visited an infectious farm (i.e., a farm in state either $T_{h}$ or $C_{h}$ ). The number of contacts by vehicles transporting products per farm per day was assumed to be Poisson distributed with a rate of 0.4 (Kartsen et al., 2005a). The probability of CSFV introduction into a farm per contact with contaminated vehicles transporting products was modeled using a Bernoulli distribution with mean 0.0068 (Stegeman et al., 2002). More precisely, at a particular day and for each farm, we computed the number of vehicles visiting a farm using the Poisson (0.4). Then, we grouped into a list the farms of a same integration group that will be visited by vehicles, we recombined this list randomly and we obtained the visit order. Finally, a vehicle was specified to visit each farm in the integration group following the visit order until the fourth trip, then we consider a new vehicle starting for the next farm in the list. An illustration of this process is explained in the following example: at a particular day, considering an integrator group compound by farms 1, 2, 4, 6, 7, 10, 11, 13, 15 and 17 and, using Poisson (0.4), we obtain that farms 1, 2, 6, 10, 13 and 15 will not receive vehicles; farms 4 and 7 will receive 1 vehicle; and farms 11 and 17 will received 2 vehicles. We create the list of farms visited by vehicles $\{4,7,11,11,17,17\}$, we recombine this list randomly and we obtain $\{4,17,11,7,11,17\}$. A first vehicle will visit the four first

201 farms in this order: 4, 17, 11 and 7 and, a second vehicle will visit farms 11 and 17.

202 Transmission of CSFV by contact of people visiting farms was assumed to occur only between farms belonging to the same $\operatorname{ADS}\left(A D S_{h}\right)$ and with the assumption that a person can visit a maximum of three farms per day (CyL expert opinion, 2008) and can only been

\footnotetext{
${ }^{\mathrm{b}}$ Expert opinion elicitation performed for Foot-and-Mouth disease and classical swine fever with the Castile and Leon veterinary services, during November $5^{\text {th }} 2008$.
} 
infectious if previously has visited an infectious farm. The number of contacts of people per farm per day was assumed to be Poisson distributed with a rate of 0.3 (Kartsen et al., 2005a).

207 The probability of CSFV introduction into a farm per contact with contaminated persons was modeled using a Bernoulli distribution with mean 0.0065 (Stegeman et al., 2002). The same process used for integration group was applied. infected farms by indirect contacts such as airborne spread or fomits. The probability of CSFV-infection by local spread from farm $h$ to farm $k$ per day, was modeled with the expression

$$
\frac{I p_{h}(t)}{\bar{N}(t)} * \operatorname{LSM}(h, k)
$$

215 where $I p_{h}(t)$ is the number of infected pigs at farm $h$ at day $t, \bar{N}(t)$ is the mean number of pigs

216 in all considered farms at day $t$ and, $\operatorname{LSM}(h, k)$ is the mean probability of infection due to local spread computed as a function of the distance between $h$ and $k$ built by interpolating the following combination of probability of infection per day and distance (meters): 0.014 at $150 \mathrm{~m}, 0.009$ at $250 \mathrm{~m}, 0.0038$ at $500 \mathrm{~m}, 0.0019$ at $1000 \mathrm{~m}$ and 0 at $2000 \mathrm{~m}$ (Kartsen et al., 2005b).

If CSFV is introduced by any of the indirect contacts from farm $h$ to farm $k$, the number of "infected" pigs in farm $k\left(I p_{k}\right)$ is increased by one.

\subsubsection{Transition from "infected" to "infectious" state}

The transition of a farm $h$ from "infected" to "infectious" state depends on the latent period that was modeled using a Poisson distribution with mean of 7 days, assuming day 0 as the day of infection in farm $h$ (Kartsen et al., 2005a). 
The transition of a farm $h$ from "infectious" to "clinical signs" state depends on the

231 incubation period that was modeled using a Poisson distribution with mean of 21 days after 232 the beginning of the infectious state in farm $h$ (Kartsen et al., 2005a).

\subsection{Detection of disease}

Detection of disease was simulated differently before and after detection of the first

infected farm (i.e. index case). Before detection of the index case, disease was assumed to be detected by observation of clinical signs on farms, whereas after detection of the index case, disease was assumed to be detected either by clinical signs or serological tests. index case was modeled using a Bernoulli distribution with mean 0.03 (Kartsen et al., 2005b).

241 After detection of the index case this probability was modeled using a Bernoulli distribution

242 with mean $0.98\left(\frac{I p_{h}(t)}{N_{h}(t)}\right)$ for farms within the control zone, $0.95\left(\frac{I p_{h}(t)}{N_{h}(t)}\right)$ for farms within the surveillance zone (CyL expert opinion, 2008) and 0.06 for farms outside control and surveillance zones (Kartsen et al., 2005b) (see Section 2.6). The probability of detection based on serological tests was assumed to consider a sample size large enough to detect a seroprevalence of $10 \%$ within the farm with a confidence of $95 \%$ (MAPA, 2006).

\subsection{Control measures}

Measures applied in the model to control disease were those defined in the European and Spanish legislation to control CSF (CyL expert opinion, 2008), which were based on zoning; restriction of movements of animals, vehicles and people; depopulation of farms detected as infected; increase of surveillance, which implies increase on the probability of 
detection of an infected farm, and; tracing of the historical ( $<60$ days) incoming or outgoing contacts in the detected farm.

\subsubsection{Zoning}

Control ( $<3 \mathrm{~km}$ radius) and surveillance ( $<10 \mathrm{~km}$ radius $)$ zones were defined around a

farm detected as infected, and movement restrictions were applied to farms within those radius during a time period of 30 and 40 days, respectively. Overlapping of control and surveillance zones after detection of new outbreaks in the area was allowed in the model.

\subsubsection{Movement restrictions}

Probability of restriction of animals, vehicle and people movements on the detected as

infected farm, were assumed to be Bernoulli distributed with a mean of $0.99,0.95$ and 0.80 ,

respectively. Those values were $0.95,0.90$ and 0.70 , respectively, for the farms within the

control and surveillance zones. Furthermore, after each detection, movement restrictions were

applied for the rest of the pig farms in the study region outside control and surveillance zones for a period of 90 days following a Bernoulli distribution with a mean of $0.4(\mathrm{CyL}$ expert opinion, 2008).

\subsubsection{Depopulation}

The depopulation of a farm detected as infected was assumed to occur after a random

273 time period with probabilities 0.11 at day $0,0.58$ at day $1,0.2$ at day $2,0.06$ at day $3,0.04$ at day 4, 0.004 at day 5, 0.003 at day 6 and 0.0015 at day 7 and 8 (Elbers et al., 1999). The maximum number of farms to be depopulated per day was assumed to be Poisson distributed with mean of 20 farms (CyL expert opinion, 2008). If this limit is reached the farm will be depopulated the day after. A depopulated farm would be not susceptible or infectious until its 
repopulation. The repopulation of the depopulated farm occurs after a period that was modeled using a Poisson distribution with a rate of 90 days (CyL expert opinion, 2008). The number of susceptible animals used to repopulate the farm was the same number of animals present on farm at the beginning of the simulation, $N_{h}(0)$.

\subsubsection{Tracing}

Tracing activities involved the process to identify contacts leaving or entering a farm detected as infected. The objective of tracing is to identify potentially infectious contacts which may have introduced disease into the farm or spread disease to other farms. The probability of tracing an animal, vehicle or people movement was assumed to be Bernoulli distributed with mean $0.99,0.70$ and 0.40 respectively (CyL expert opinion, 2008). Tracing was applied for animal, vehicle and people contacts occurring <60 days before detection of the infected farm, with a probability to escape from this control that follows a Bernoulli law with mean 0.01 (CyL expert opinion, 2008). Serological test were used to detect disease in all traced farms. The maximum number of farms to be traced per day was assumed to be Poisson distributed with mean of 60 farms (CyL expert opinion, 2008). If this limit is reached the farm will be traced the day after.

\subsection{Model experimentation}

Segovia, one of the most important areas of pig production in Spain which was affected by the 1997-1998 CSF-epidemic in Spain and for which data were available, was used to illustrate the model performance. Registration of pig farms and notification of animal movements is mandatory by the Spanish legislation, so all pig farms and pig movements in Segovia region were assumed to be registered. During 2008, Segovia registered 1,400 pig farms and 1,108,415 pigs, and there were 10,468 movements. The $49.6 \%$ of the farms were 
303

304

305

306

307

308

310

311

312

314

315

316

317

318

classified as farrow-to-finish, $36.4 \%$ fattening, $13.6 \%$ farrowing and $0.35 \%$ artificial insemination centres. CSF is an exotic disease for the Spanish pig population and no CSF vaccination is performed into the country. Therefore, it was assumed that all pig farms were susceptible to CSFV infection (i.e. naive population). The input variables of the model were estimated using real data provided by the Regional Government of Castile and Leon (CyL) and the expert opinion of CyL Veterinary Services obtained in November 5, 2008.

We consider two experiments, one without control measures, denoted by NM and the other with all control measures described in section 2.6. and denoted by WM. The maximum time interval in the NM experiment was set to $T=100$ days, whereas no maximum time interval was set for the WM experiment. In both cases, we run 1000 simulations.

The model and the statistical analysis were performed using MatLab interface (Ver.7.5.0.342 R2007b-The MathWorks Inc.) on a Pentium 4 of $3.4 \mathrm{Ghz}$ with $2 \mathrm{~Gb}$ and needs 64000 seconds for the NM case and 20000 seconds for the WM case. Maps were developed using ArcGIS 9.2 (ESRI).

\subsection{Model verification and sensitivity analysis}

The experiments with and without control measures were repeated several times to assure consistency of results. Furthermore, the maximum time interval in the experiment without control measures was changed to $\mathrm{T}=200$ and $\mathrm{T}=365$ and the dynamic of the epidemic was evaluated.

The large number of input variables used in the model formulation and the little information available on many of these variables make necessary the application of an extensive and detailed sensitivity analysis to identify the influence of each variable on the model outcomes. This is the reason why a complete sensitivity analysis, together with validation of the model using data from the 1997-1998 CSFV-epidemic in Segovia, will be presented in a second 
manuscript entitled "A novel spatial and stochastic model to evaluate the within and between farm transmission of classical swine fever virus: II. Sensitivity analysis and validation" (to be submitted). Nevertheless, in this paper we present a preliminary sensitivity analysis to test the overall sensitivity of the model and to evaluate the value of input variables for which uncertainty may be high (those obtained by expert opinion). Those inputs obtained by expert opinion were systematically varied from minimum and maximum values equal to, respectively, a $10 \%$ reduction and a $10 \%$ increase in the base value of the variable, while keeping constant on their base values all other variables. A percentage of change smaller than $10 \%$ in outputs was considered evidence of lack of influence of input variables in model results.

\section{Results}

\subsection{Within-farm transmission}

The mean spread of CSFV within a farm reaching the infectious state (i.e. after the latent period) was observed to occur very fast, mainly for the farrowing farms (Figure 1). As a result, $80 \%$ of the animals were infected at day 8,11 and 23 for the farrowing, farrow-tofinish and fattening farms, respectively.

\subsection{Magnitude and duration of CSFV epidemic without control measures}

The estimated mean and 95\% PI of infected farms in the experiment without control measures were $7[1,33], 32[1,138]$ and $234[2,705]$ for the simulations with maximum time interval of 100, 200 and 365 days, respectively. The main sources of transmission were local spread and vehicles transporting products (feed, slurry, etc) which were responsible of $62.4 \%$ 
$353[0,100]$ and $21.1 \%[0,100]$ of the infections, respectively. The movement of people, animal

354 movements and vehicles transporting animals were responsible of the $9.6 \%[0,85.6], 6.8 \%$

$355[0,50]$ and $0.1 \%[0,1]$ of the infections, respectively.

\subsection{Magnitude and duration of CSFV epidemic with control measures}

The estimated mean and 95\% PI number of infected farms, quarantined farms in the

control zone and quarantined farms in the surveillance zone in the experiment with control

measures were $3[1,17], 23[0,76]$ and $115[0,318]$, respectively. The peak of infections is reached at day 47 (Figure 2). The duration of the epidemic was 63 [26, 177] days. $[0,100]$ of the infections. Other ways of transmission were the movement of vehicles transporting products $(21.3 \%,[0,100])$, movement of animals $(8.8 \%,[0,100])$, people contacts $(8.5 \%,[0,100])$ and vehicles transporting animals $(0.1 \%,[0,1])$.

The median distance (meters) from an infected farm to the 10 nearest farms was 1.64 times smaller $($ median $=436,95 \%$ PI $[20 ; 2485])$ than the distance from a non infected farm to the 10 nearest farms $($ median $=713,95 \%$ PI $[711,719])$.

The mean basic reproduction ratios $\left(R_{0}\right)$ for farrowing, farrow-to-finish and fattening

371 farms were 2.98 [0-15.25], 3.43[0-19.00], 1.72 [0-10.00], respectively. The mean effective 372 reproduction ratios $(R)$ were 2.05 [0-12.5], 1.79 [0-10.55], 1.33 [0-8.00], respectively. Risk

373 values for those farms were $1.66[0,8.25], 1.72[0,8.00]$ and $1.62[0,7.00]$, respectively.

374 Artificial insemination centres registered an $R_{0}$ and $R$ of 0.2 [0, 0.9], and a risk value of 0.8

$375[0,1.9]$. Slaughterhouses had an $R_{0}$ and $R$ of $0.05[0,0.12]$, and a risk value of 89.52 [0, 376 398.20]. Spatial distribution of the effective reproduction ratio and risk values for Segovia 377 region is presented in Figure 3. 
Model results were not very sensitive to changes in input parameters $(<10 \%$ of change). Only $1 \%$ of the simulations present an increase in the maximum duration of the epidemic (those lasting more than 600 days) (Figure 2).

383

\section{Discussion}

The Be-FAST model presented here allows to stochastically simulate the potential within- and between- farms spread of CSFV, to quantify the magnitude and duration of a CSFV epidemic and to identify areas at high risk for the introduction and spread of the disease.

This model incorporates two important improvements compared with other previous models based on InterSpread and described by Karsten et al., (2005a,b) and Jalvingh et al., (1999).

The first one is the ability to explicitly model the within-farm CSFV spread, which

393 also modulates the between-farm transmission of the virus. Traditionally, the infectivity within a farm was considered to be constant from the day of infection till depopulation of the farm. However, the number of animals infected on the farm changes over time, due to withinfarm spread and, consequently, the probability of a farm to transmit CSFV to other farms by

397 direct or indirect contacts also changes. Moreover, the within-spread is usually different

398 depending on the type of farm considered, because modifications in structure and demographics within the farm substantially change the contact patterns among individuals

400 (Klinkenberg et al., 2002). The model presented here is the first one that incorporates the 401 explicit simulation of the within-farm spread dynamics in the different types of pig farms 402 (Figure 1). 
The second improvement is the introduction of the real farm-to-farm contact network.

404 Previous studies assumed a mean number of contacts for all farms, without considering the

405 farm size or the production system on farm (Karsten et al., 2005a). The model presented here

406 distinguishes not only the contacts patterns between different types of farms (i.e. farrowing,

407 farrow-to-finish, fattening and artificial insemination centers), like in the study of Jalvingh et

408 al., (1999), but also, the specific individual farm-to-farm trade patterns.

Two other differences of the Be-FAST disease model are the explicit consideration of

the limited resources for depopulation measures and the repopulation of farms after

411 depopulation/disinfection. Most of the models assumed that all infected farms would be

412 depopulated the day after detection of infection, no matter the number of farms to be

413 depopulated. Similarly, no repopulation of the depopulated farms was assumed during the epidemic. These assumptions, which seem reasonable for small epidemics, may not be realistic in large epidemics, where capacities for slaughtering will be exceed, delaying the time to depopulation and where depopulated farms will be repopulated increasing the susceptible population. The consequence of those simplifications is an underestimation of the magnitude and duration of the simulated epidemics, and may certainly impact the reliability of the model results, as it has been discussed by Karsten et al. (2005a) and Jalvingh et al., (1999).

Model results showed a relative small magnitude and duration of the epidemic in the scenario with control measures, with an average of 3 infected farms and 63 days of epidemic duration, which was similar to the 1-5 infected farms and 21-60 of duration obtained by Karsten et al. (2005a). 
most of pig farms in Segovia region are located in high density areas. Movements of animals from farm-to-farm helped to introduce the virus in remote CSFV-free areas, hindering the control of the epidemic.

Areas with high number of incoming infections (i.e. Risk of introduction) were related to those areas with high number of outgoing infections (i.e. effective reproduction ratio) (Figure 3). Furthermore, those areas were relatively constant in both the experiment withoutand with- control measures, although with an important reduction in the experiment with control measures. These findings involve that the risk of introduction and spread of disease is concentrated in the same regions and, therefore, allocation of preventive and control measures in those areas may allow to meaningfully reduce the number of infections. Other interesting result was the high risk value of the slaughterhouses (Risk= 89.52). This implies that slaughterhouses were likely to receive high number of infected animals during an epidemic and may be good places to centralize efforts to increase sensitivity of the surveillance programs in a CSFV epidemic. Spatial and stochastic models are complex and time consuming from a computational 443 point of view; however, results usually are more realistic and adjusted to the region of study, offering a useful tool for policy makers. One of the most important shortcomings when developing complex epidemic models is the data quality and the potential high computational time needed to run the simulations and obtain the stochastic results. Fortunately, for this

447 study high quality data was provided by Castile and Leon region, allowing to explicitly 448 include into the model the farm demographics (i.e. production type, farm size), farm location 449 (geographical coordinates) and the complex time-space network of direct and indirect contacts in the study region. However, in order to reduce the high computational time needed to obtain results, it was also necessary to implement some simplifications and assumptions in 452 the model. Maybe one of the most important simplifications was to simulate the latent period 
453 (i.e. transition between infected to infectious) at a farm level instead of at the animal level.

454 This assumption allowed to simplify the within-herd transmission process by using an SI

455 (Susceptible-Infectious) instead of the more complex SEIR (Susceptible-Exposed-Infectious-

456 Removed) model. Various simulations were run to compare both approaches (using the SI

457 and the SEIR within-farm model), and results showed that the SI allowed to reduce almost

$45840 \%$ of the computation time without significantly affecting ( $<5 \%$ change) the model

459 outcomes. Because time is very important for decision making when using a model as a

460 decision support tool during an epidemic, authors decide to keep the simple SI within-farm

461 spread model to reduce the computational time.

462 The parameterization of the model was based on the epidemiological characteristics of

463 the disease obtained from previous studies and expert opinion of the Regional Animal Health

464 Authorities. Most of the parameterization was based on scientific publications related to

465 recent outbreaks in regions which circumstances and conditions were similar to the Spanish

466 ones (Kartsen et al., 2005a,b; Klinkenberg et al., 2002; Stegeman et al., 2002). However, no

467 published data was available for some parameters such as restriction of movements, capacity

468 of depopulation per time period, time to repopulation and, the probability of tracing a

469 movement, and expert opinion was needed to obtain values that could be adapted to the study

470 region. Because the value of the input parameters, particularly those obtained by expert

471 opinion, may have associated high variability and uncertainty, sensitivity analysis was

472 conducted to specifically quantify the nature and magnitude of change in the outcomes when

473 varying those initial values. Sensitivity analysis demonstrated the overall robustness of the

474 parameters used in the Be-FAST model, showing that variations in the values of the

475 parameters evaluated did not significantly $(<10 \%)$ affect model outcomes (Figure 2).

476 Nevertheless, the performance of every single parameter and their influence on the model

477 outcomes will be presented in detail in a future manuscript, as well as the influence of aspects 
such as farm size or farm location (i.e. high/low animal density areas). Validation using data

479

480

481

482

483

484

485

486

487

488

489

490

491

492

493

494

495

496

497

498

499

500

501

502 from 1997-1998 CSFV-epidemic in Segovia region of Spain will be also presented in detail in this future publication.

The methods and results presented in this study may be useful for developing programs and contingency plans for the prevention and control of CSFV in Segovia. However, the results of simulations of the model must always be interpreted with caution for making decisions. The assumptions and the uncertainties associated with some model parameters should be evaluated as well as the influence that the unpredictability of human behavior can have on the network of contacts and business relationships. It should be considered that the results presented here, with data from Segovia, are indicative of the performance of the model, and in no case should be extrapolated directly to other Spanish regions or countries, where demographic conditions, contact patterns and management of the epidemic may substantially differ. However, the transparent nature of the model, with clear definition of each parameter and the values used, makes easy to adapt it to other regions or countries, after some appropriate adjustments on the input values. Furthermore, it would be interesting to instigate collaborations with other international research groups in order to have access to unpublished data and to study a potential broader application of the model.

Future research would be focus on the evaluation of the current and alternative (such as vaccination) control measures to be applied and the economical impact of a CSFVepidemic.

\section{Conclusion}

The model described here allows to stochastically simulate the potential within- and between- farms spread of CSFV, considering the spatial heterogeneities and the real farm-to- 
503 farm contact network into a specific region. Methods and results presented here may be

504 useful for the decision making process and for the improvement of the prevention and control

505 programmes for CSFV.

506

507 Acknowledgements

508

509

The project was funded in part by the project MTM2008-04621 of the Spanish

510 Ministry of Science and Innovation, the Universidad Complutense de Madrid (Research

511 group 910480), the Regional Government of Castile and León Region (JCyL) and the

512 Spanish Ministry of Environment and Rural and Marine Affairs (MARM). We gratefully

513 acknowledge the assistance of Olga Minguez and her team (JCyL) for providing data and

514 assistance in the interpretation of the results and the two anonymous reviewers for their

515 useful comments and suggestions.

516

517 Conflict of interest

518 None of the authors of this manuscript has personal or financial relationship with

519 people or organizations that could influence or bias the work and results presented in this 520 paper.

521

522 References

523 Anderson, R.M., May, R.M., 1979. Population biology of infectious diseases: Part I. Nature. $280,361-367$.

Anderson, R.M., May, R.M., 1991. Infectious Diseases of Humans. Oxford, U.K.: Oxford University Press. 
Crauwels, A.P.P.; Nielen, M.; Elbers, A.R.W.; Stegeman, J.A.; Tielen, M.J.M., 2003. Neighbourhood infections of classical swine fever during the 1997-1998 epidemic in the Netherlands. Prev. Vet. Med., 61, 263-277.

De Vos, C.J., Saatkamp, H.W., Huirne, R.B., Dijkhuizen, A.A., 2003. The risk of the introduction of classical swine fever virus at regional level in the European Union: a conceptual framework. Rev. Sci. Tech., 22, 795-810.

Elbers, A.T.W., Stegeman, A., Moser, H., Ekker, H.M., Smak, J.A., Pluimers, H., 1999. The classical swine fever epidemic 1997-1998 in the Netherlands: descriptive epidemiology. Prev. Vet. Med., 42, 157-184.

Jalvingh, A.W., Nielen, M., Maurice, H., Stegeman, A.J., Elbers A.R.W., Dijkhuizen, A.A., 1999. Spatial and stochastic simulation to evaluate the impact of events and control measures on the 1997-1998 classical swine fever epidemic in The Netherlands. I. Description of simulation model, Vet. Microbiol., 42, 271-295.

Kartsen, S., Rave, G., Krieter, J., 2005a. Monte Calro simulation of classical swine fever epidemics and control I. General concepts and description of the model. Vet. Microbiol., 108, 187-198.

Kartsen, S., Rave, G., Krieter, J., 2005b. Monte Calro simulation of classical swine fever epidemics and control II. Validation of the model. Vet. Microbiol. 108, 187-198.

Klinkenberg, D., De Bree, J., Laevens, H., De Jong, M.C.M., 2002. Within- and Between-pen transmission of Classical Swine Fever Virus: a new method to estimate the basic reproduction ration from transmission experiments. Epidemiol. Infect., 128, 293-299.

MAPA, 2006, Manual práctico de operaciones en la lucho contra la peste porcina clásica (PPC). 121 pages. Available at: http://rasve.mapa.es/Publica/InformacionGeneral/Documentos/Manuales/Manual\%20 PPC\%202006.pdf 
Martínez-López, B., Perez, A.M., Sánchez-Vizcaíno, J.M., 2009. Evaluation of the potential

553

554

555

556

557

558

559

560 spread and effectiveness of control measures for Classical Swine Fever into Spain by using a spatial and stochastic model. 12th International Symposium on Veterinary Epidemiology and Economics (ISVEE), 10-14 August, 2009, Durban, South Africa.

Milne, G., Fermanis, C., Johnston, P., 2008. A mobility model for classical swine fever in feral pig populations. Vet. Res., 39, 53.

OIE 2009b. WAHID. Available at: http://www.oie.int/wahis/public.php?page=home OIE, 2009a. Handistatus II. Available at: http://www.oie.int/hs2/report.asp?lang=en

Ribbens, S., Dewulf, J., Koenen, F., Maes, D., de Kruif, A., 2007. Evidence of indirect transmission of classical swine fever virus through contacts with people. Vet Rec.,160, 687-690.

Saatkamp, H.W., Dijkhuizen, A.A., Geers, R., Huirne R.B.M., Noordhuizent, J.P.T.M., Goedseels, V., 1996a. Simulation studies on the epidemiological impact of national identification and recording systems on the control of classical swine fever in Belgium. Prev. Vet. Med., 26, 119-132

Stärk, K.D.C., 1998. Systems for the prevention and control of infectious diseases in pigs. $\mathrm{PhD}$ thesis. EpiCentre. Massey University, New Zealand.

Stegeman, A., Elbers, A.R.W., Smak, J., de Jong, M.C.M., 1999. Quantification of the transmission of classical swine fever virus between farms during the 1997-1998 epidemic in The Netherlands. Prev. Vet. Med., 42, 219-234.

Stegeman, J.A., Elbers, A.R.W., Bouma, A., De Jong, M.C.M., 2002. Rate of inter-farm transmission of classical swine fever virus by different types of contact during the 1997-8 epidemic in The Netherlands. Epidemiol. Infect., 128, 285-291. 


\section{Table and Figure captions}

577

578 Table 1. Assumptions and parameters used within the Be-FAST CSFV-model.

579

580 Figure 1. Dynamic of within-farm CSFV-transmission in the fattening, farrow-to-finish and farrowing farms.

582

583 Figure 2. Epidemic curve showing the range of the number of infected farms per day in the

584 experiment with control measures (dark grey) and when $10 \%$ of perturbation of the input

585 variables is applied (light grey).

586

587 Figure 3. Spatial distribution of the incoming infections (i.e. Risk of introduction) (TOP) and

588 outgoing infections (i.e. effective reproduction ratio) (BOTTOM) obtained in the Be-FAST

589 CSFV-model in Segovia region for the experiment without control measures (LEFT) and

590 with control measures (RIGHT). Therefore, Figure 3a represents the spatial distribution of the

591 incoming infections without control measures, Figure $3 \mathrm{~b}$ the incoming infections with control

592 measures, Figure $3 \mathrm{c}$ the outgoing infections without control measures and Figure $3 \mathrm{~d}$ the

593 outgoing infections with control measures. 
Table 1.

\section{Parameter}

Within-farm transmission parameter for farrowing pig farms

Within-farm transmission parameter for fattening pig farms

Within-farm transmission parameter for farrow-to-finish pig farms

Number of pig movements from farm $h$ to farm $k$ at day $t$

Number of pigs moved during a simulated movement from farm $h$ to farm $k$ at day $t$

Probability of infection by animal contacts due to a movement from farm $h$ to farm $k$ at day $t$

Probability of infection by contact with vehicles transporting infected animals

Maximum number of visits that a vehicle can do per day

Number of contacts with vehicles transporting products per farm at day $t$

Probability of infection by contacts with vehicles transporting products

Maximum number of visits that a person can do per day

Number of contacts with people per farm at day $t$

Probability of infection by contact with people

Probability of infection by local spread at day $t$

Latent period (transition from infected to infectious state)

Transition from infectious to clinical signs state
Value

$\beta_{h}=8.52$

$\beta_{h}=1.85$

$\beta_{h}=5.18$

Poisson $[M(t)]^{*} m_{h k} / M T^{(1)}$

$n_{h k} * N_{h}(t) / N_{h}(0){ }^{(2)}$

Poisson $[M(t)]^{*} m_{h k} / M T * n_{h k}$ $* I p_{h}(t) / N_{h}(0)^{(3)}$

Bernoulli [0.011]

4

Poisson [0.4]

Bernoulli [0.0068]

3

Poisson [0.3]

Bernoulli [0.0065]

$\frac{I p_{h}(t)}{\bar{N}(t)} * \operatorname{LSM}(h, k)$

Poisson [7]

Poisson [21]

\section{Reference}

Klinkenberg et al., 2002

Klinkenberg et al., 2002

Klinkenberg et al., 2002

CyL data

CyL data

Klinkenberg et al., 2002

Stegeman et al., 2002

CyL expert opinion, 2008

Kartsen et al., 2005a

Stegeman et al., 2002

CyL expert opinion, 2008

Kartsen et al., 2005a

Stegeman et al., 2002

Kartsen et al., 2005b

Kartsen et al., 2005a

Kartsen et al., 2005a 
Probability of detection based on clinical signs at day $t$ before detection of the index case

Probability of detection based on clinical signs at day $t$ outside the control and

surveillance zones

Probability of detection based on clinical signs at day $t$ in the control zone

Probability of detection based on clinical signs at day $t$ in the surveillance zone

Probability of detection based on serological test

Radius (duration) of the control zone

Radius (duration) of the surveillance zone

Probability of restriction of animal movements on the detected as infected farm

Probability of restriction of vehicle movements on the detected as infected farm

Probability of restriction of people movements on the detected as infected farm

Probability of restriction of animal movements within the control and surveillance zone

Probability of restriction of vehicle movements within the control and surveillance zone

Probability of restriction of people movements within the control and surveillance zone

Duration of the restriction of movements outside the control and surveillance zones

Probability of restriction of movements outside the control and surveillance zones

Probability to depopulate a detected as infected farm

\section{Bernoulli [0.03] \\ Bernoulli [0.06]}

Bernoulli $\left[0.98\left(\frac{I p_{h}(t)}{N_{h}(t)}\right)\right]$

Bernoulli $\left[0.95\left(\frac{I p_{h}(t)}{N_{h}(t)}\right)\right]$

Bernoulli [0.95]

$3 \mathrm{~km}$ (30 days)

$10 \mathrm{~km}$ (40 days)

Bernoulli [0.99]

Bernoulli [0.95]

Bernoulli [0.80]

Bernoulli [0.95]

Bernoulli [0.90]

Bernoulli [0.70]

90 days

Bernoulli [0.4]

Table [prob,day]: [0.11,0],

$[0.58,1],[0.2,2],[0.06,3]$,
Kartsen et al., 2005b

Kartsen et al., 2005b

CyL expert opinion, 2008

CyL expert opinion, 2008

MAPA, 2006

MAPA, 2006

MAPA, 2006

CyL expert opinion, 2008

CyL expert opinion, 2008

CyL expert opinion, 2008

CyL expert opinion, 2008

CyL expert opinion, 2008

CyL expert opinion, 2008

CyL expert opinion, 2008

CyL expert opinion, 2008

Elbers et al., 1999 
Maximum number of farms to be depopulated at day $t$

Time to repopulation of a depopulated farm

Probability of tracing an animal movement

Probability of tracing a vehicle movement

Probability of tracing a people movement

Period of time to consider when tracing historical incoming or outgoing contacts

Probability of not tracing an animal, vehicle or people movement

Maximum number of farms to be traced at day $t$

\section{$[0.04,4],[0.004,5],[0.003,6]$,}

$[0.0015,7]$ and $[0.0015,8]$

Poisson [20]

CyL expert opinion, 2008

Poisson [90]

CyL expert opinion, 2008

Bernoulli [0.99]

Bernoulli [0.70]

Bernoulli [0.40]

60 days

Bernoulli [0.01]

Poisson [60]
CyL expert opinion, 2008

CyL expert opinion, 2008

CyL expert opinion, 2008

CyL expert opinion, 2008

CyL expert opinion, 2008

CyL expert opinion, 2008

${ }^{(1)} M(t)=$ number of movements occurring during 2008 at day $t ; m_{h k}=$ number of movements from farm $h$ to farm $k$ during $2008 ; M T=$ total number of movements in the study region during 2008

${ }^{(2)} n_{h k}=$ number of pigs moved between farm $h$ and $k$ during 2008; $N_{h}(t)=$ number of pigs on farm $h$ at time $t$.

${ }^{(3)} I p_{h}(t)=$ number of infected pigs on farm $h$ at time $t$. 


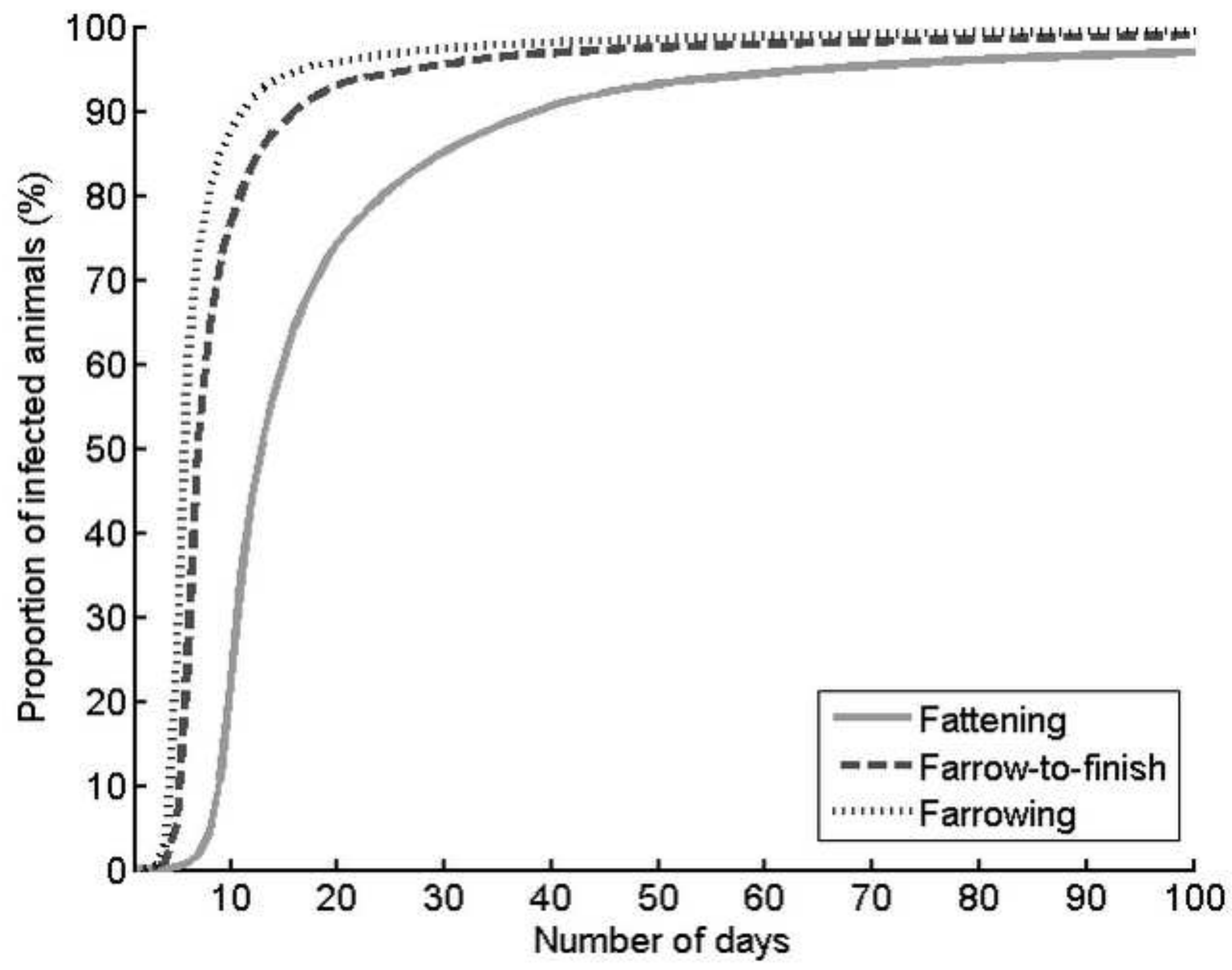




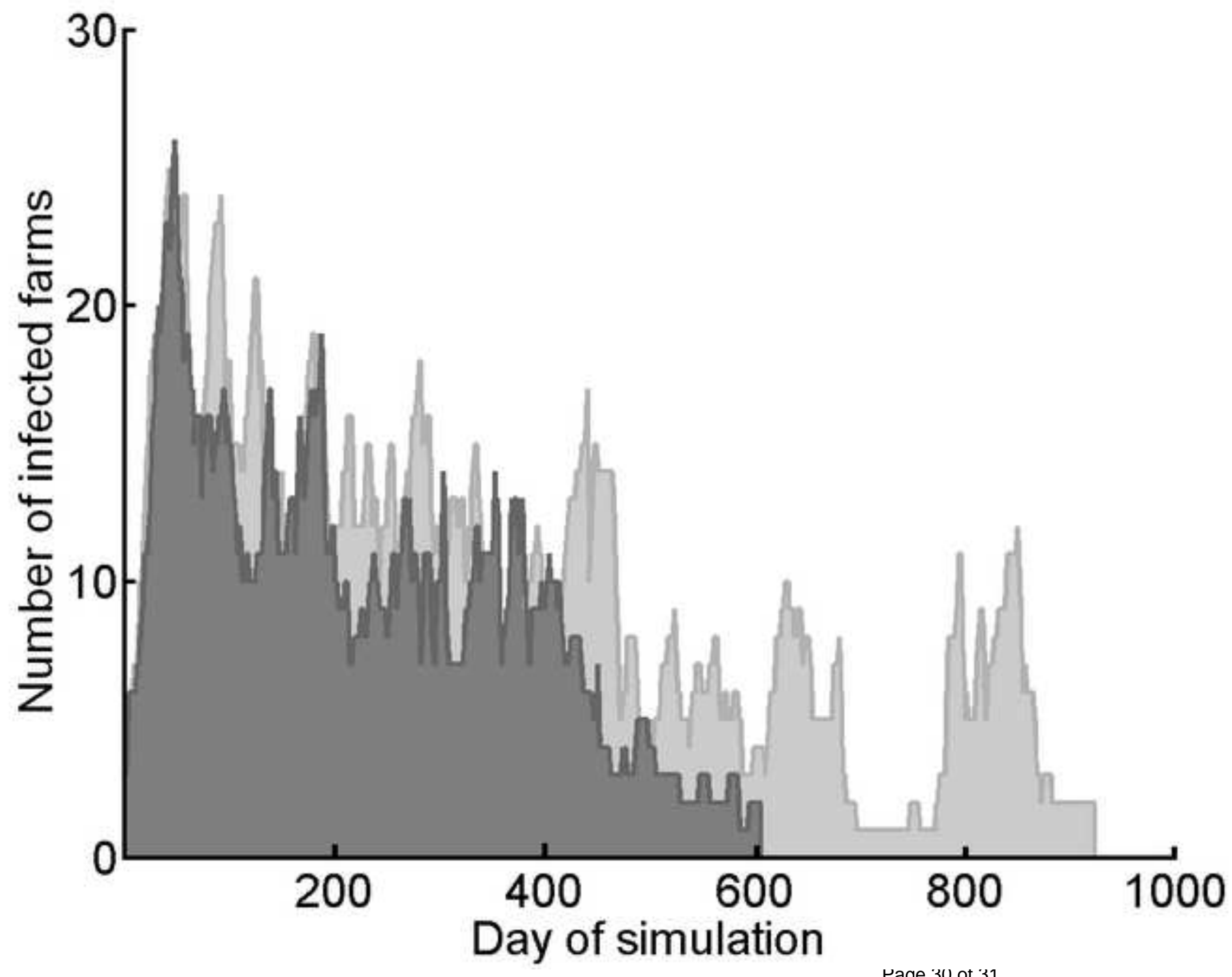




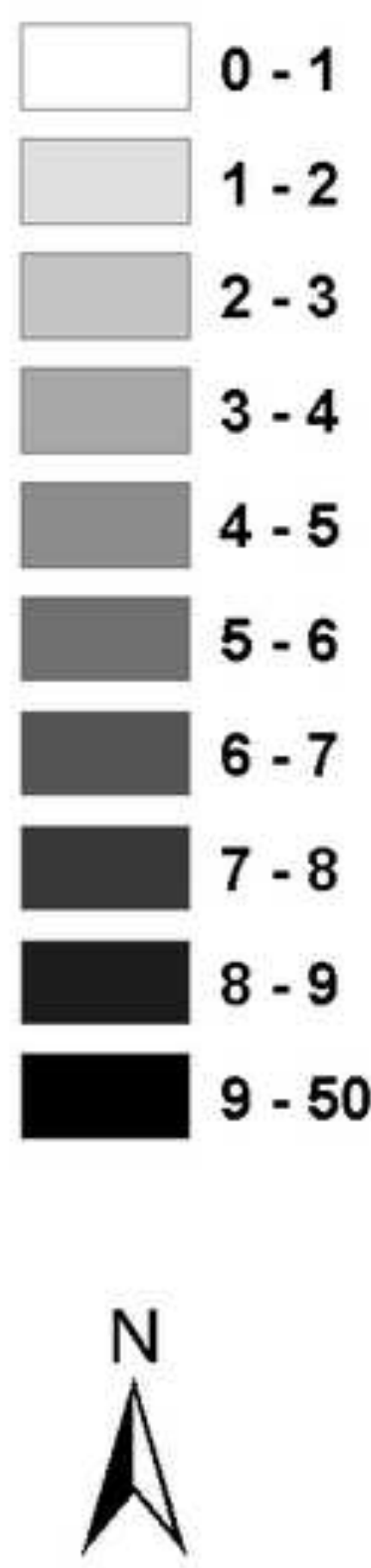

(a)

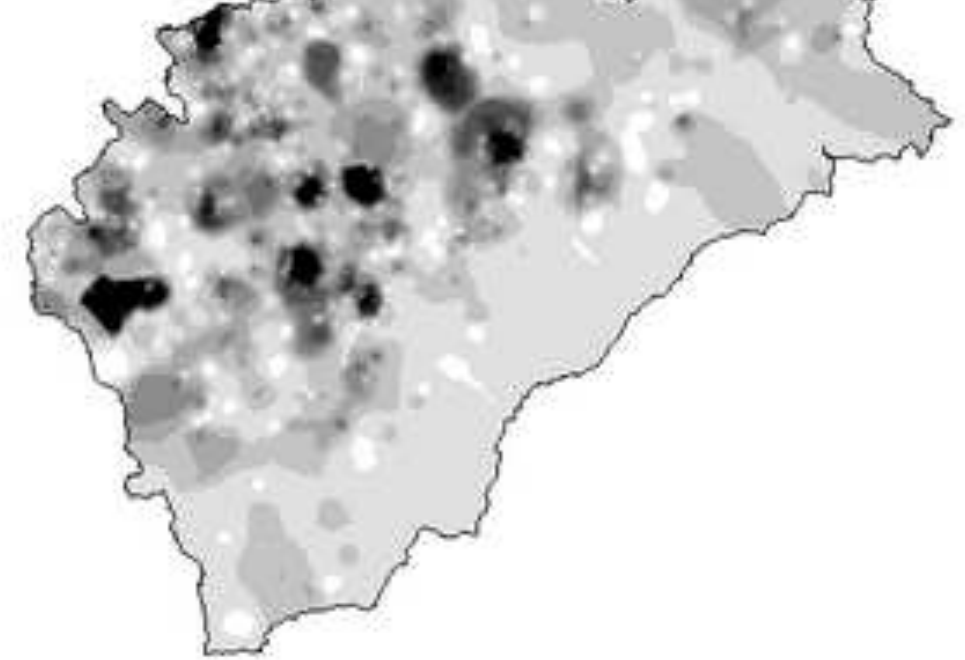

(c)

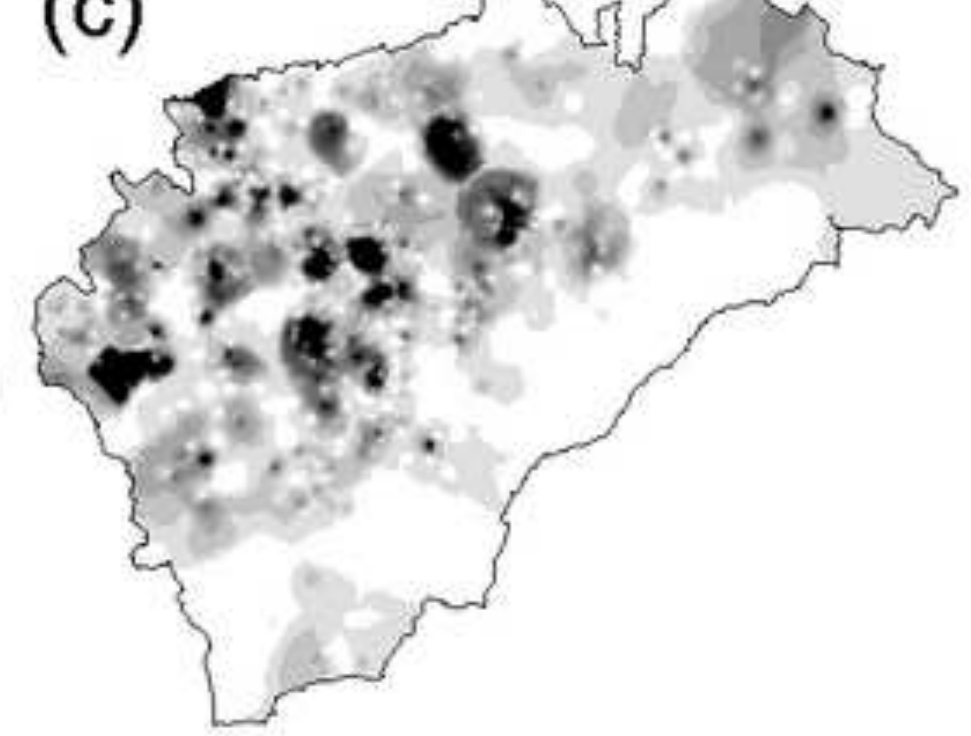

(b)

(d)

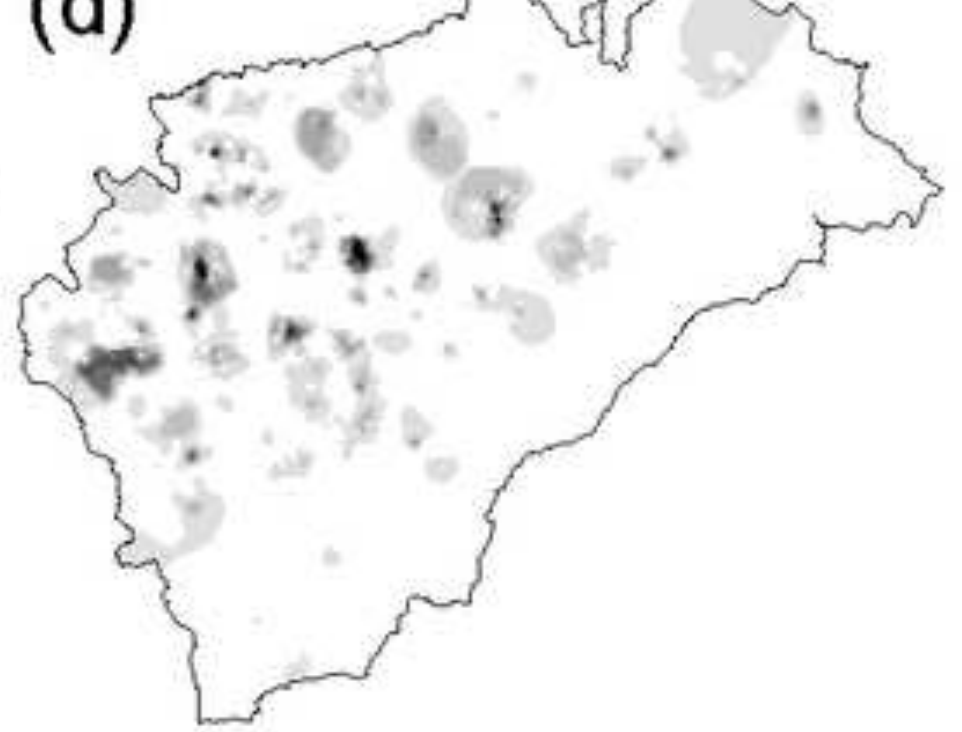

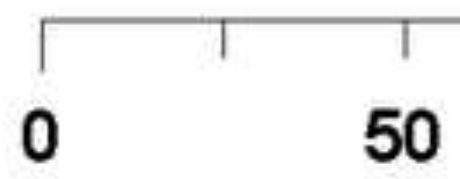
100 Kilometers 